\title{
Turbo MIMO-OFDM Receiver in Time-Varying Channels
}

\author{
Yu-Kuan Chang, Fang-Biau Ueng and Yi-Wei Jhang \\ Department of Electrical Engineering \\ National Chung-Hsing University \\ Taichung, Taiwan \\ Email: fbueng@nchu.edu.tw \\ *Corresponding author: Fang-Biau Ueng
}

Received October 16, 2017; revised December 11, 2017; accepted January 21, 2018; published August 31, 2018

\begin{abstract}
This paper proposes an advanced turbo receiver with joint inter-carrier interference (ICI) self cancellation and channel equalization for multiple-input multiple-output orthogonal frequency division multiplexing (MIMO-OFDM) systems over rapidly time-varying channel environment. The ICI caused by impairment of local oscillators and carrier frequency offset (CFO) is the major problem for MIMO-OFDM communication systems. The existing schemes (conjugate cancellation (CC) and phase rotated conjugate cancellation (PRCC)) that deal with the ICI cancellation and channel equalization can't provide satisfactory performance over time-varying channels. In term of error rate performance and low computational complexity, ICI self cancellation is the best choice. So, this paper proposes a turbo receiver to deal with the problem of joint ICI self cancellation and channel equalization. We employ the adaptive phase rotations in the receiver to effectively track the CFO variations without feeding back the CFO estimate to the transmitter as required in traditional existing scheme. We also give some simulations to verify the proposed scheme. The proposed schene outperforms the existing schemes.
\end{abstract}

Keywords: MIMO-OFDM, inter-carrier interference, ICI self-cancellation, channel estimation

This work was supported in part by the Ministry of Science and Technology of Taiwan under Contract MOST 106-2221-E-005-047. 


\section{Introduction}

The MIMO transmission system is employed to increase the transmitted data rate and improve the communication link quality compared with single-antenna system [1]-[3]. Furthermore, the Space-Time Block codes provides temporal/spatial multiplexing and have been employed to enhance the reliability of MIMO wireless communication systems and improve the performance of MIMO communications [4]. The OFDM technique can effectively combat the problem of frequency selective fading and can provide the advantages of high-data-rate transmission and high spectral efficiency [5]-[6]. Many wireless standards have employed the OFDM technique [7]-[8]. Therefore, for this reason, the MIMO-OFDM technique exploits the advantages of the MIMO and OFDM and has been a promising technique for future wireless systems. The main problem of the MIMO-OFDM system is that the system is highly sensitive to the CFO.

In order to effectively deal with the problem of the CFO, the estimation the CFO must be derived and the CFO compensation is then performed that is according to the estimated results [9]-[11]. For OFDM communication systems, based on the assumption that the channel variation is with approximate linearity in an OFDM symbol, [12]-[14] proposed the ICI cancellation schemes that use training sequences and pilot symbols to perform frequency-domain channel estimation/equalization over fast fading multipath channels. Also, in the literatures, some techniques without employing pilot symbols or training sequences is available to cancel ICI. Among these schemes, the ICI self-cancellation approaches have attracted much attention. The ICI self-cancellation approaches have the characteristic that the ICI can be cancelled in the reception procedure without performing complicated equalization or inserting extra symbols. The conjugate cancellation (CC) [15] and phase rotated conjugate cancellation (PRCC) [16] are the two most important ICI self-cancellation schemes. The existing schemes (conjugate cancellation (CC) [15] and phase rotated conjugate cancellation (PRCC) [16]) that deal with the ICI cancellation and channel equalization can't provide satisfactory performance over time-varying channels. For the CC scheme, the first path transmits the original OFDM symbol, and the second path transmits the conjugate version of the OFDM symbol. Based on the configuration, we find that the ICI in the first path is restrained by the second path. For the PRCC scheme, by applying a phase rotation to the transmitter in the CC scheme, we find that the ICI caused by both paths can be mutually cancelled more effectively. The existing CC and PRCC schemes can't work well in time-varying channels where the CFOs changes fast with time. Moreover, the existing PRCC scheme must feedback the estimation of CFO to the transmitter to obtain the optimal phase rotation. Therefore, the PRCC scheme needs additional signal overhead.

In this paper, we propose a turbo adaptive receiver that can overcome the above mentioned problem. Furthermore, the existing two-stage IQ imbalance scheme [17] uses a two-stage procedure to estimate and compensate the CFO and the IQ imbalance for MIMO-OFDM communications. Although the two-stage IQ imbalance scheme proposes a new CFO compensation method to cancel the ICI for the MIMO-OFDM communications, the channel equalization and channel estimation that can improve the bit error rate performance have not been considered. The phase error problem is also not considered in the two-stage IQ imbalance scheme. So the two-stage IQ imbalance scheme can't provide satisfactory performance under environments with large CFOs. We propose a turbo receiver for ICI 
self-cancellation by taking advantage of conjugate transmission [18] for the OFDM communications. Unlike the existing PRCC method, the proposed scheme uses two phase rotations on both paths at the receiver to cope with the fast fading channel. Furthermore, in order to obtain the formula of between the CFO and the phase rotation, we also derive the optimal phase rotations based in the maximization of the carrier-to-interference ratio (CIR), and also propose an adaptive process employing the normalized block least mean-squared (BLMS) algorithm [19]-[21]. Therefore, the requirement of feeding back the estimation of CFO information can be eliminated. [22] proposed a precoding-based blind channel estimation for MIMO-OFDM systems. This scheme doesn't solve the problem of the CFO and can't be applied to the time-varying channel. [23] proposed a decision-directed channel estimation scheme to deal with the shortage of pilot for the MIMO-OFDM systems [23]. To practically analyze and effectively solve the CFO problem of MIMO-OFDM systems over time-varying channels, this paper joint considers the channel estimation/equalization based on the Kalman algorithm [24] [25], turbo minimum mean square error (MMSE) equalization [26] and the CFO compensation that employs an adaptive modified PRCC receiver [18]. The proposed Kalman algorithm belongs to the pilot-symbol-aided parametric channel estimation method in which the channel responses are characterized as a collection of sparse propagation paths. Based on the signal subspace of the channel samples' correlation matrix, the estimation of channel parameters can be translated into an unconstrained minimization problem. Then, in order to solve this optimization problem, a subspace tracking by Kalman filter is carried out which is characterized in the state equation and the measurement equation. Turbo equalization is joint equalization and decoding and had been proposed for MIMO OFDM systems [27]-[29]. [30] deals with frequency-domain equalization for faster-than-Nyquist (FTN) signaling in doubly selective channels (DSCs). [31] studies the receiver design for a coded faster-than-Nyquist signaling system in time-variant intersymbol interference channels. In this paper, we design a turbo receiver that is joint Kalman channel estimation, CFO compensation and turbo equalization for MIMO OFDM system over time-varying channels. The proposed schene outperforms the existing schemes. The organization of this paper is as follows. In section 2, the system model, the existing CC scheme and the existing PRCC scheme are described. The proposed scheme is described in section 3, the detailed frequency offset estimation scheme, the Kalman channel estimation algorithm and Turbo equalization are also given in section 3. Finally, some simulation results and conclusions are provided in section 4 and section 5, respectively.

\section{System Model}

The considered MIMO-OFDM transmitter is described as Fig. 1. The proposed receiver is described as Fig. 2. The time-domain received signals of the two paths can be expressed as follows,

$$
\begin{aligned}
& \mathbf{y}_{\alpha, l, n}^{(1)}=\sum_{\beta=1}^{N_{t}} \sum_{p=0}^{L-1} d_{\beta,(l-p), n} h_{\beta, \alpha, p, n} e^{j \frac{2 \pi}{N} l \varepsilon_{\alpha, n}}+w_{\alpha, l, n}^{(1)} \quad l=0,1, \cdots, N-1 \\
& \mathbf{y}_{\alpha, l, n}^{(2)}=\sum_{\beta=1}^{N_{t}} \sum_{p=0}^{L-1} d_{\beta,(l-p), n}^{*} h_{\beta, \alpha, p, n} e^{j \frac{2 \pi}{N} l \varepsilon_{\alpha, n}}+w_{\alpha, l, n}^{(2)} \quad l=0,1, \cdots, N-1
\end{aligned}
$$




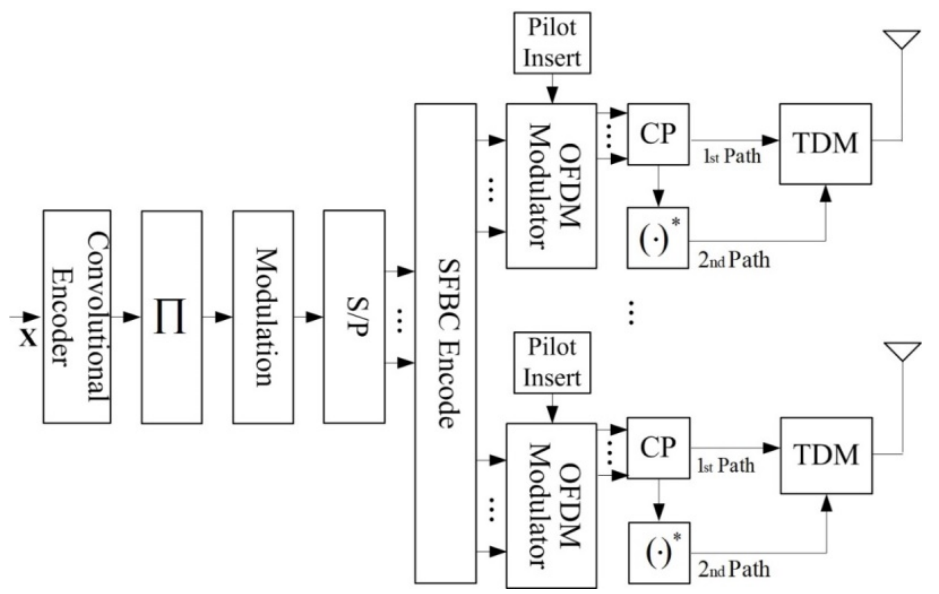

Fig. 1. The transmitter.

where $L$ is the number of channel tap, $\mathbf{y}_{\alpha, l, n}^{(i)}$ and $w_{\alpha, l, n}^{(i)}$ correspond to the received symbol and noise at the $\alpha$-th receiving antenna, $l$-th subcarrier in the $i$-th path within the $n$-th transmission block before the FFT demodulation, respectively. $h_{\beta, \alpha, p, n}$ corresponds to the channel tap at the $\beta$-th transmitting antenna, $\alpha$-th receiving antenna and $p$-th path within the $n$-th transmission block, and then $\beta=$ $1, \ldots, N_{t}$ and $\alpha=1, \ldots, N_{r}, N_{t}$ is the total number of transmitting antenna, $N_{r}$ is the total number of receiving antenna. The channel taps vary per block. If we employ $2 \times 2 \mathrm{STBC}$ encoder that $d_{1, l, n}$ is the $2 \times 2$ STBC encoder output at the first antenna in the $l$-th subcarrier within the $n$-th transmission block, we obtain

$$
\left[\begin{array}{l}
d_{1, l, n} \\
d_{2, l, n}
\end{array}\right]=\left[\begin{array}{ccccc}
x_{0} & -x_{1}{ }^{*} & \cdots & x_{N-2} & -x_{N-1}{ }^{*} \\
x_{1} & x_{0}{ }^{*} & \cdots & x_{N-1} & x_{N-2}{ }^{*}
\end{array}\right]
$$

The frequency-domain received signals of these two paths can be written as follows,

$$
\begin{aligned}
& \mathbf{Y}_{\alpha, m, n}^{(1)}=\frac{\mathbf{1}}{\sqrt{N}} \sum_{l=0}^{N-1}\left(\mathbf{y}_{\alpha, l, n}^{(1)}\right) \mathrm{e}^{-j \frac{2 \pi}{N} l m} \\
& =\frac{\mathbf{1}}{\sqrt{N}} \sum_{l=0}^{N-1}\left(\sum_{\beta=1}^{N_{t}} \sum_{p=0}^{L-1} d_{\beta,(l-p), n} h_{\alpha, \beta, p, n} e^{j \frac{2 \pi}{N} l \varepsilon_{\alpha, n}}+w_{\alpha, l, n}^{(1)}\right) \mathrm{e}^{-j \frac{2 \pi}{N} l m} \\
& =\frac{1}{\sqrt{N}} \sum_{l=0}^{N-1} \sum_{\beta=1}^{N_{t}}\left(\frac{1}{\sqrt{N}} \sum_{i=0}^{N-1}\left[D_{\beta, i, n} e^{j \frac{2 \pi}{N}(l-p) i}\left(\sum_{p=0}^{L-1} h_{\alpha, \beta, p, n} e^{j \frac{2 \pi}{N} i p}\right) \mathrm{e}^{j \frac{2 \pi}{N} l \varepsilon_{\alpha, n}}\right]\right. \\
& \left.+w_{\alpha, l, n}^{(1)}\right) e^{-j \frac{2 \pi}{N} l m} \\
& =\sum_{\beta=1}^{N_{t}} \sum_{i=0}^{N-1} D_{\beta, i, n}\left[\frac{1}{N} \sum_{l=0}^{N-1}\left(\sum_{p=0}^{L-1} h_{\alpha, \beta, p, n} e^{j \frac{2 \pi}{N} i p}\right) e^{-j \frac{2 \pi}{N}\left(i+\varepsilon_{\alpha, n}-l m\right)}\right] \\
& +\frac{1}{\sqrt{N}} \sum_{l=0}^{N-1} w_{\alpha, l, n}^{(1)} e^{-j \frac{2 \pi}{N} l m} \\
& =D_{\beta, m, n} H_{\alpha, \beta, p, n} \mathrm{C}\left(-\varepsilon_{\alpha, n}\right)+\sum_{\substack{i=0 \\
i \neq m}}^{N-1} D_{N_{t}, i, n} H_{N_{t}, N_{r}, i, n} \mathrm{C}\left(\mathrm{m}-\mathrm{i}-\varepsilon_{\alpha, n}\right)+W_{\alpha, m, n}^{(1)}, \\
& \mathrm{m}=0,1, \cdots, N-1
\end{aligned}
$$




$$
\begin{aligned}
\mathbf{Y}_{\alpha, m, n}^{(2)}=\frac{\mathbf{1}}{\sqrt{N}} \sum_{l=0}^{N-1} & \left(\mathbf{y}_{\alpha, l, n}^{(2)}\right)^{*} \mathrm{e}^{-j \frac{2 \pi}{N} l m} \\
& =\frac{1}{\sqrt{N}} \sum_{l=0}^{N-1}\left(\sum_{p=0}^{L-1} d_{\beta,(l-p), n}^{*} h_{\alpha, \beta, p, n} e^{j \frac{2 \pi}{N} l\left(\varepsilon_{\alpha, n}+\Delta \varepsilon_{\alpha, n}\right)}+w_{\alpha, l, n}^{(2)}\right)^{*} e^{-j \frac{2 \pi}{N} l m} \\
& =D_{\beta, m, n} H_{\alpha, \beta, p, n} \mathrm{C}\left(\varepsilon_{\alpha, n}+\Delta \varepsilon_{\alpha, n}\right) \\
& +\sum_{\substack{i=0 \\
i=m}}^{N-1} D_{\beta, i, n} H_{\alpha, \beta, i, n} \mathrm{C}\left(\mathrm{m}-\mathrm{i}+\left(\varepsilon_{\alpha, n}+\Delta \varepsilon_{\alpha, n}\right)\right)+W_{\alpha, m, n}^{(2)} \\
& m=0,1, \cdots, N-1
\end{aligned}
$$

where $D_{\beta, m, n}$ is the FFT of $2 \times 2$ STBC encoder output. By combining and averaging the signal processed in (4) and (5), and define $C(v)=\frac{1}{N} \sum_{n=0}^{N-1} e^{-j \frac{2 \pi}{N} n v}$, we obtain

$$
\begin{aligned}
\mathbf{R}_{\alpha, m, n}=\frac{1}{2}\left(\mathbf{Y}_{\alpha, m, n}^{(1)}\right. & \left.+\mathbf{Y}_{\alpha, m, n}^{(2)}\right) \\
& =\frac{1}{2}\left[e^{j \varphi_{\alpha, n}} C\left(-\varepsilon_{\alpha, n}\right)+e^{-j \varphi_{\alpha, n}} C\left(+\varepsilon_{\alpha, n}\right)\right] \sum_{\beta=1}^{N_{t}} D_{\beta, m, n} H_{\beta, \alpha, m, n} \\
& +\frac{1}{2} \sum_{\substack{i=0 \\
i \neq m}}^{N-1}\left[e^{j \varphi_{\alpha, n}} C\left(m-i-\varepsilon_{\alpha, n}\right)+e^{-j \varphi_{\alpha, n}} C\left(m-i+\varepsilon_{\alpha, n}\right)\right] D_{\beta, m, n} H_{\beta, \alpha, m, n} \\
& +\frac{1}{2}\left[W_{\alpha, m, n}^{(1)}+W_{\alpha, m, n}^{(2)}\right], \quad m=0,1, \cdots, N-1
\end{aligned}
$$

The CIR of the CC scheme in 2x2 MIMO-OFDM can be defined as

$$
C I R_{\alpha, c c}=\frac{\left|C\left(-\varepsilon_{\alpha, n}\right)+C\left(\varepsilon_{\alpha, n}\right)\right|^{2}}{\sum_{i=0}^{N-1}\left|C\left(i-\varepsilon_{\alpha, n}\right)+C\left(i+\varepsilon_{\alpha, n}\right)\right|^{2}}
$$

For the PRCC scheme, the first path transmits the original MIMO-OFDM signals with phase rotation $\varphi_{\alpha, n}$, while the second path transmits the conjugate version of the original MIMO-OFDM signals with phase rotation $-\varphi_{\alpha, n}$, i.e., the $d_{\beta, l, n} e^{j \varphi_{\alpha, n}}$ signal is transmitted in the first path and the $\left\{d_{\beta, l, n} e^{-j \varphi_{\alpha, n}}\right\}^{*}$ is transmitted in the second path, respectively. Therefore, the time-domain received signals of the two paths can be expressed as follows,

$$
\begin{aligned}
& \mathbf{y}_{\alpha, l, n}^{(1)}=\sum_{\beta=1}^{N_{t}} \sum_{p=0}^{L-1} d_{\beta,(l-p), n} h_{\beta, \alpha, p, n} e^{j \varphi_{\alpha, n}} e^{j \frac{2 \pi}{N} l \varepsilon_{\alpha, n}}+w_{\alpha, l, n}^{(1)}, \quad l=0,1, \cdots, N-1 \\
& \mathbf{y}_{\alpha, l, n}^{(2)}=\sum_{p=0}^{L-1} d_{\beta,(l-p), n}^{*} h_{\beta, \alpha, p, n} e^{j \varphi_{\alpha, n}} e^{j \frac{2 \pi}{N} l \varepsilon_{\alpha, n}}+w_{\alpha, l, n}^{(2)}, \quad l=0,1, \cdots, N-1
\end{aligned}
$$

And the frequency-domain received signals of the two paths can be written as follows,

$$
\mathbf{Y}_{\alpha, m, n}^{(1)}=\frac{\mathbf{1}}{\sqrt{N}} \sum_{l=0}^{N-1}\left(\mathbf{y}_{\alpha, l, n}^{(1)}\right) \mathrm{e}^{-j \frac{2 \pi}{N} l m}
$$




$$
\begin{aligned}
& =\frac{\mathbf{1}}{\sqrt{N}} \sum_{l=0}^{N-1}\left(\sum_{\beta=1}^{N_{t}} \sum_{p=0}^{L-1} d_{\beta,(l-p), n} h_{\alpha, \beta, p, n} e^{j \emptyset_{\alpha, n}} e^{j \frac{2 \pi}{N} l \varepsilon_{\alpha, n}}+w_{\alpha, l, n}^{(1)}\right) \mathrm{e}^{-j \frac{2 \pi}{N} l m} \\
& =\frac{1}{\sqrt{N}} \sum_{l=0}^{N-1} \sum_{\beta=1}^{N_{t}}\left(\frac{1}{\sqrt{N}} \sum_{i=0}^{N-1}\left[D_{\beta, i, n} e^{j \frac{2 \pi}{N} l i}\left(\sum_{p=0}^{L-1} h_{\alpha, \beta, p, n} e^{j \frac{2 \pi}{N} i p}\right) e^{j \phi_{\alpha, n}} \mathrm{e}^{j \frac{2 \pi}{N} l \varepsilon_{\alpha, n}}\right]+w_{\alpha, l, n}^{(1)}\right) e^{-j \frac{2 \pi}{N} l m} \\
& =\sum_{\beta=1}^{N_{t}} \sum_{i=0}^{N-1} D_{\beta, i, n}\left[\frac{1}{N} \sum_{l=0}^{N-1}\left(\sum_{p=0}^{L-1} h_{\alpha, \beta, p, n} e^{j \frac{2 \pi}{N} i p}\right) e^{j \phi_{\alpha, n}} e^{-j \frac{2 \pi}{N}\left(m-i-\varepsilon_{\alpha, n}\right)}\right]+\frac{1}{\sqrt{N}} \sum_{l=0}^{N-1} w_{\alpha, l, n}^{(1)} e^{-j \frac{2 \pi}{N} l n} \\
& =D_{\beta, m, n} H_{\alpha, \beta, p, n} e^{j \phi_{\alpha, n}} \mathrm{C}\left(-\varepsilon_{\alpha, n}\right)+\sum_{\substack{i=0 \\
i \neq m}}^{N-1} D_{N_{t}, i, n} H_{\alpha, \beta, i, n} e^{j \phi_{\alpha, n}} \mathrm{C}\left(\mathrm{m}-\mathrm{i}-\varepsilon_{\alpha, n}\right)+W_{\alpha, m, n}^{(1)}, \\
& m=0,1, \cdots, N-1 \\
& \mathbf{Y}_{\alpha, m, n}^{(2)}=\frac{\mathbf{1}}{\sqrt{N}} \sum_{l=0}^{N-1}\left(\mathbf{y}_{\alpha, l, n}^{(2)}\right)^{*} e^{-j \phi_{\alpha, n}} \mathrm{e}^{-j \frac{2 \pi}{N} l m} \\
& =\frac{1}{\sqrt{N}} \sum_{l=0}^{N-1}\left(\sum_{p=0}^{L-1} d_{\beta,(l-p), n}^{*} h_{\alpha, \beta, p, n} \varepsilon_{\alpha, n} e^{j \frac{2 \pi}{N} l \varepsilon_{\alpha, n}}+w_{\alpha, l, n}^{(2)}\right)^{*} e^{-j \frac{2 \pi}{N} l m} \\
& =D_{\beta, m, n} H_{\alpha, \beta, p, n} e^{-j \phi_{\alpha, n}} \mathrm{C}\left(\varepsilon_{\alpha, n}\right)+\sum_{\substack{i=0 \\
i \neq m}}^{N-1} D_{\beta, i, n} H_{\alpha, \beta, i, n} \mathrm{C}\left(m-i+\varepsilon_{\alpha, n}\right)+W_{\alpha, m, n}^{(2)} \\
& m=0,1, \cdots, N-1
\end{aligned}
$$

By combining and averaging the signal processed in (10) and (11), we obtain

$$
\begin{aligned}
\mathbf{R}_{\alpha, m, n}=\frac{1}{2}\left(\mathbf{Y}_{\alpha, m, n}^{(1)}+\right. & \left.\mathbf{Y}_{\alpha, m, n}^{(2)}\right) \\
= & \frac{1}{2}\left[e^{j \varphi_{\alpha, n}} C\left(-\varepsilon_{\alpha, n}\right)+e^{-j \varphi_{\alpha, n}} C\left(+\varepsilon_{N_{r}, n}\right)\right] \sum_{\beta=1}^{N_{t}} D_{\beta, m, n} H_{\beta, \alpha, m, n} \\
& +\frac{1}{2} \sum_{\substack{i=0 \\
i \neq m}}^{N-1}\left[e^{j \varphi_{\alpha, n}} C\left(m-i-\varepsilon_{\alpha, n}\right)+e^{-j \varphi_{\alpha, n}} C\left(m-i+\varepsilon_{\alpha, n}\right)\right] D_{\beta, m, n} H_{\beta, \alpha, m, n} \\
& +\frac{1}{2}\left[W_{\alpha, m, n}^{(1)}+W_{\alpha, m, n}^{(2)}\right], \quad m=0,1, \cdots, N-1
\end{aligned}
$$

From (12), the CIR of the PRCC scheme in 2x2 MIMO-OFDM can be defined as

$$
C I R_{\alpha, p r c c}=\frac{\left|e^{j \varphi_{\alpha, n}} C\left(-\varepsilon_{\alpha, n}\right)+e^{-j \varphi_{\alpha, n}} C\left(\varepsilon_{\alpha, n}\right)\right|^{2}}{\sum_{i=0}^{N-1}\left|e^{j \varphi_{\alpha, n}} C\left(i-\varepsilon_{\alpha, n}\right)+e^{-j \varphi_{\alpha, n}} C\left(i+\varepsilon_{\alpha, n}\right)\right|^{2}}
$$

We can optimize $\varphi_{\alpha, n}$ in terms of maximizing the CIR in (13),

$$
\varphi_{\alpha, n}^{(o p t)}=\operatorname{argmax} C I R_{P R C C}\left(\varepsilon_{\alpha, n}, \varphi_{\alpha, n}\right)
$$

The optimal phase rotation can be express as follows

$$
\varphi_{\alpha, n}^{(o p t)}=-\pi \varepsilon_{\alpha, n} \frac{N-1}{N}
$$

\section{The Proposed Turbo Receiver}

The proposed turbo receiver for MIMO-OFDM system is shown as Fig. 2. With the presence of frequency offset $\varepsilon_{\alpha, n}$, the time-domain received signals from the two transmission paths can be described as follows, 


$$
\begin{gathered}
\mathbf{y}_{\alpha, l, n}^{(1)}=\sum_{\beta=1}^{N_{t}} \sum_{p=0}^{L-1} d_{\beta,(l-p), n} h_{\beta, \alpha, p, n} e^{j \frac{2 \pi}{N} l \varepsilon_{\alpha, n}}+w_{\alpha, l, n}^{(1)} l=0,1, \cdots, N-1 \\
\mathbf{y}_{\alpha, l, n}^{(2)}=\sum_{\beta=1}^{N_{t}} \sum_{p=0}^{L-1} d_{\beta,(l-p), n}^{*} h_{\beta, \alpha, p, n} e^{j \frac{2 \pi}{N} l\left(\varepsilon_{\alpha, n}+\Delta \varepsilon_{\alpha, n}\right)}+w_{\alpha, l, n}^{(2)} l=0,1, \cdots, N-1
\end{gathered}
$$

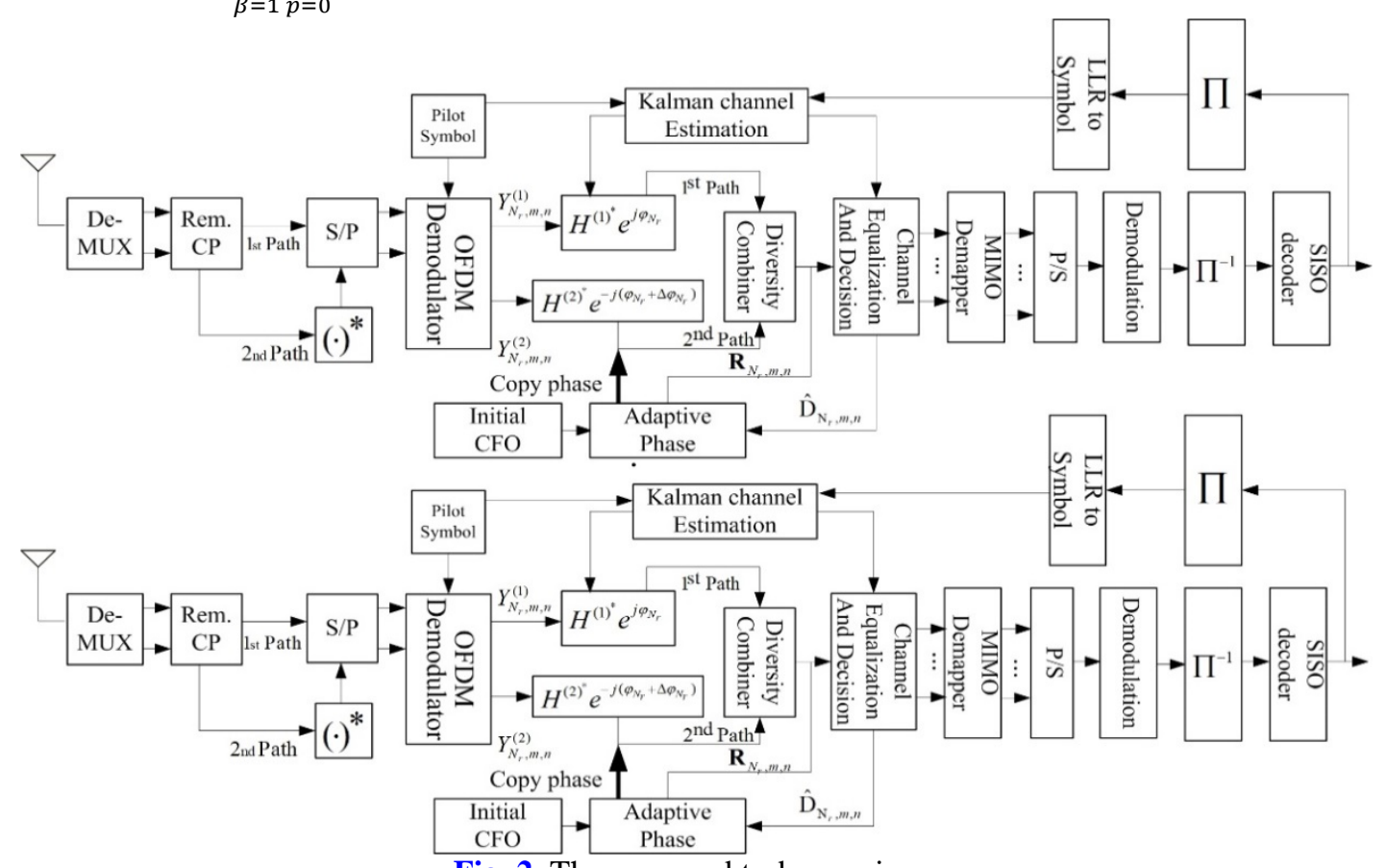

Fig. 2. The proposed turbo receiver.

After performing FFT operation, we obtain the following frequency-domain signals as follows,

$$
\begin{aligned}
& \mathbf{Y}_{\alpha, m, n}^{(1)}=\frac{\mathbf{1}}{\sqrt{N}} \sum_{l=0}^{N-1}\left(\mathbf{y}_{\alpha, l, n}^{(1)}\right) \mathrm{e}^{-j \frac{2 \pi}{N} l m} \\
& =\frac{\mathbf{1}}{\sqrt{N}} \sum_{l=0}^{N-1}\left(\sum_{\beta=1}^{N_{t}} \sum_{p=0}^{L-1} d_{\beta,(l-p), n} h_{\alpha, \beta, p, n} e^{j \frac{2 \pi}{N} l \varepsilon_{\alpha, n}}+w_{\alpha, l, n}^{(1)}\right) \mathrm{e}^{-j \frac{2 \pi}{N} l m} \\
& =\frac{1}{\sqrt{N}} \sum_{l=0}^{N-1} \sum_{\beta=1}^{N_{t}}\left(\frac{1}{\sqrt{N}} \sum_{i=0}^{N-1}\left[D_{\beta, i, n} e^{j \frac{2 \pi}{N} l i}\left(\sum_{p=0}^{L-1} h_{\alpha, \beta, p, n} e^{j \frac{2 \pi}{N} i p}\right) \mathrm{e}^{j \frac{2 \pi}{N} l \varepsilon_{\alpha, n}}\right]+w_{\alpha, l, n}^{(1)}\right) e^{-j \frac{2 \pi}{N} l m} \\
& =\sum_{\beta=1}^{N_{t}} \sum_{i=0}^{N-1} D_{\beta, i, n}\left[\frac{1}{N} \sum_{l=0}^{N-1}\left(\sum_{p=0}^{L-1} h_{\alpha, \beta, p, n} e^{j \frac{2 \pi}{N} i p}\right) e^{-j \frac{2 \pi}{N}\left(m-i-\varepsilon_{\alpha, n}\right)}\right]+\frac{1}{\sqrt{N}} \sum_{l=0}^{N-1} w_{\alpha, l, n}^{(1)} e^{-j \frac{2 \pi}{N} l n} \\
& =D_{\beta, m, n} H_{\alpha, \beta, p, n} e^{j \emptyset_{\alpha, n}} \mathrm{C}\left(-\varepsilon_{\alpha, n}\right)+\sum_{\beta=1}^{N_{t}} \sum_{\substack{i=0 \\
i \neq m}}^{N-1} D_{N_{t}, i, n} H_{\alpha, \beta, i, n} e^{j \phi_{\alpha, n}} \mathrm{C}\left(m-\mathrm{i}-\varepsilon_{\alpha, n}\right)+W_{\alpha, m, n}^{(1)}, \\
& m=0,1, \cdots, N-1 \\
& \mathbf{Y}_{\alpha, m, n}^{(2)}=D_{\beta, m, n} H_{\alpha, \beta, p, n} \mathrm{C}\left(\varepsilon_{\alpha, n}+\Delta \varepsilon_{\alpha, n}\right)+\sum_{\beta=1}^{N_{t}} \sum_{\substack{i=0 \\
i \neq m}}^{N-1} D_{\beta, i, n} H_{\alpha, \beta, i, n} \mathrm{C}\left(m-i+\varepsilon_{\alpha, n}+\right. \\
& \left.\Delta \varepsilon_{\alpha, n}\right)+W_{\alpha, m, n}^{(2)}, m=0,1, \cdots, N-1
\end{aligned}
$$


where $\mathbf{Y}_{\alpha, m, n}^{(i)}, H_{\alpha, \beta, p, n}$ and $W_{\alpha, m, n}^{(i)}$ correspond to the received symbol, channel response and noise at the $\beta$-th transmitting antenna, the $\alpha$-th receiving antenna, the $m$-th subcarrier in the $i$-th path within the $n$-th transmission after FFT modulation, respectively. $\varepsilon_{\alpha, n}$ and $\varepsilon_{\alpha, n}+$ $\Delta \varepsilon_{\alpha, n}$ denote the CFOs in the two paths at the $\alpha$-th receiving antenna within the $n$-th transmission, respectively. After performing the individual phase rotations and the weighting coefficients, the signals in both paths can be written as follows,

$$
\begin{gathered}
\mathbf{R}_{\alpha, m, n}^{(1)}=e^{j \varphi_{\alpha, n}} \mathbf{H}_{\beta, \alpha, m, n}^{(1) *} \mathbf{Y}_{\alpha, m, n}^{(1)} \\
\mathbf{R}_{\alpha, m, n}^{(2)}=e^{j\left(\varphi_{\alpha, n}+\Delta \varphi_{\alpha, n}\right)} \mathbf{H}_{\beta, \alpha, m, n}^{(2) *} \mathbf{Y}_{\alpha, m, n}^{(2)}
\end{gathered}
$$

where $\varphi_{\alpha, n}$ and $\varphi_{\alpha, n}+\Delta \varphi_{\alpha, n}$ denote the designed phase rotations in the two paths at the $\alpha$-th receiving antenna within the $n$-th transmission, respectively. Finally, the output signal can be expressed as follows,

$$
\begin{aligned}
\mathbf{R}_{\alpha, m, n}=\frac{1}{2}\left(\mathbf{Y}_{\alpha, m, n}^{(1)}\right. & \left.+\mathbf{Y}_{\alpha, m, n}^{(2)}\right) \\
& =\frac{1}{2} \sum_{\beta=1}^{N_{t}}\left(D_{\beta, m, n}\left|H_{\beta, \alpha, m, n}^{(1)}\right|^{2} e^{j \varphi_{\alpha, n}} C\left(-\varepsilon_{\alpha, n}\right)\right. \\
& \left.+D_{\beta, m, n}\left|H_{\beta, \alpha, m, n}^{(2)}\right|^{2} e^{-j \varphi_{\alpha, n}+\Delta \varphi_{N_{r}, n}} C\left(-\varepsilon_{\alpha, n}+\Delta \varepsilon_{N_{r}, n}\right)\right) \\
& +\sum_{l=0}^{N-1}\left[D_{\beta, l, n} H_{\beta, \alpha, m, n}^{(1) *} H_{\beta, \alpha, m, n}^{(1)} e^{j \varphi_{N_{r}, n}} C\left(m-l-\varepsilon_{\alpha, n}\right)\right. \\
& \left.+D_{\beta, l, n} H_{\beta, \alpha, m, n}^{(2) *} H_{\beta, \alpha, m, n}^{(2)} e^{-j \varphi_{\alpha, n}+\Delta \varphi_{\alpha, n}} C\left(m-l+\left(\varepsilon_{\alpha, n}+\Delta \varepsilon_{\alpha, n}\right)\right)\right] \\
& \left.+\left(e^{j \varphi_{\alpha, n}} H_{\beta, \alpha, m, n}^{(1)} W_{\alpha, m, n}^{(1)}+e^{-j\left(\varphi_{N_{r}, n}+\Delta \varphi_{N_{r}, n}\right)} H_{\beta, \alpha, m, n}^{(2) *} W_{\alpha, m, n}^{(2)}\right)\right)
\end{aligned}
$$

From (22), the CIR of the proposed adaptive modified PRCC scheme can be defined as follows,

$$
\begin{aligned}
\operatorname{CIR}_{\alpha, \text { proposed }}\left(\varepsilon_{\alpha, n}, \Delta \varepsilon_{\alpha, n}, \varphi_{\alpha, n}, \Delta \varphi_{\alpha, n}\right) \\
=\frac{\left|e^{j \varphi_{\alpha, n}} C\left(-\varepsilon_{\alpha, n}\right)+e^{-j\left(\varphi_{\alpha, n}+\Delta \varphi_{\alpha, n}\right)} C\left(\left(-\varepsilon_{\alpha, n}+\Delta \varepsilon_{\alpha, n}\right)\right)\right|^{2}}{\sum_{l=1}^{N-1}\left|e^{j \varphi_{\alpha, n}} C\left(l-\varepsilon_{\alpha, n}\right)+e^{-j\left(\varphi_{\alpha, n}+\Delta \varphi_{\alpha, n}\right)} C\left(l+\left(\varepsilon_{\alpha, n}+\Delta \varepsilon_{\alpha, n}\right)\right)\right|^{2}}
\end{aligned}
$$

Given $\varepsilon_{\alpha, n}$ and $\Delta \varepsilon_{\alpha, n}$, the optimal phase rotations can be determined by maximizing the CIR,

$$
\left(\varphi_{\alpha, n}^{(o p t)}, \Delta \varphi_{\alpha, n}^{(o p t)}\right)=\operatorname{argmax} C I R_{\alpha, P R C C}\left(\varepsilon_{\alpha, n}, \Delta \varepsilon_{\alpha, n}, \varphi_{\alpha, n}, \Delta \varphi_{\alpha, n}\right)
$$

By solving (24), we can derive the following sufficient condition for the optimal phase rotations,

$$
2 \varphi_{\alpha, n}^{(o p t)}+\Delta \varphi_{\alpha, n}^{(o p t)}=-\pi \frac{\left(2 \varepsilon_{\alpha}+\Delta \varepsilon_{\alpha}\right)(N-1)}{N}
$$

So the corresponding solution is as follows,

$$
\begin{aligned}
\varphi_{\alpha, n}^{(o p t)} & =-\pi \frac{\varepsilon_{\alpha, n}(N-1)}{N} \\
\Delta \varphi_{\alpha, n}^{(o p t)} & =-\pi \frac{\Delta \varepsilon_{\alpha, n}(N-1)}{N}
\end{aligned}
$$


Assuming that the phase rotations $\varphi_{\alpha, n}$ and $\Delta \varphi_{\alpha, n}$ have approached the optimal values after sufficient adaptations, the CFOs can be estimated as follows,

$$
\begin{aligned}
\hat{\varepsilon}_{\alpha, n} & =-\varphi_{\alpha, n} \frac{N}{\pi(N-1)} \\
\Delta \hat{\varepsilon}_{\alpha, n} & =-\Delta \varphi_{\alpha, n} \frac{N}{\pi(N-1)}
\end{aligned}
$$

\subsection{The Cost Function for the Phase Update}

The equalization output based on the MMSE criteria can be expressed as follows,

$$
\widehat{\mathbf{D}}_{\alpha, m, n}=\left(\left(\mathbf{H}_{\beta, \alpha, m, n} \mathbf{H}_{\beta, \alpha, m, n}^{H}+\mathbf{I} \sigma^{2}\right)^{-1} \mathbf{H}_{\beta, \alpha, m, n}^{H}\right) \mathbf{R}_{\alpha, m, n}
$$

Then, the desired signal can be written as

$$
\mathbf{d r}_{\alpha, m, n}=e^{j \varphi_{\alpha, n}} \widehat{\mathbf{D}}_{\alpha, m, n} e^{-j\left(\hat{\varepsilon}_{\alpha, n}\right) n / N}
$$

Therefore, we can define the error signal as follows,

$$
\mathbf{e}_{\alpha, m, n}=\mathbf{d} \mathbf{r}_{\alpha, m, n}-\mathbf{R}_{\alpha, m, n}
$$

If we average the squared error signals of all the subcarriers, the cost function used in the receiver can be defined as follows,

$$
J_{\alpha, n}\left(\emptyset_{\alpha, n}, \Delta \emptyset_{\alpha, n}, \hat{\varepsilon}_{\alpha, n}, \Delta \hat{\varepsilon}_{\alpha, n}\right) \equiv \boldsymbol{E}\left\{\left|\mathbf{e}_{\alpha, m, n}\right|^{2}\right\} \cong \frac{1}{N} \sum_{m=0}^{N-1}\left|\mathbf{e}_{\alpha, m, n}\right|^{2}
$$

The phase rotation update equation employing normalized block least mean squared (BLMS) algorithm can be described as follows,

$$
\left[\begin{array}{c}
\emptyset_{\alpha, n+1} \\
\Delta \emptyset_{\alpha, n+1}
\end{array}\right]=\left[\begin{array}{c}
\emptyset_{\alpha, n} \\
\Delta \emptyset_{\alpha, n}
\end{array}\right]+\left[\begin{array}{cc}
\mu_{1} & 0 \\
0 & \mu_{2}
\end{array}\right] \frac{\nabla J\left(\emptyset_{\alpha, n} \Delta \emptyset_{\alpha, n}, \hat{\varepsilon}_{\alpha, n}, \Delta \hat{\varepsilon}_{\alpha, n}\right)}{\xi+E\left\{\mathbf{Y}_{\alpha, n}^{(1) H} \mathbf{Y}_{\alpha, n}^{(1)}+\mathbf{Y}_{\alpha, n}^{(2) H} \mathbf{Y}_{\alpha, n}^{(2)}\right\}}
$$

where $\mathbf{Y}_{\alpha, n}^{(i)}=\left[\begin{array}{llll}Y_{\alpha, 1, n}^{(i)} & Y_{\alpha, 2, n}^{(i)} & \cdots & Y_{\alpha, N, n}^{(i)}\end{array}\right]^{T}, \mu_{i}$ is the step size employed in the $i$-th paths, $\xi$ is a small positive constant.

\subsection{Kalman Channel Estimation}

Fig. 3 shows the block diagram of Kalman channel estimation in the proposed receiver. We first serve the mean of the transmitted symbol $\overline{\mathbf{X}}=\mathbf{E}[\mathbf{X}]$ as the measurement matrix $\mathbf{M}_{n}$.. In fact, we should set the received signal $\mathbf{Y}=\overline{\mathbf{X}} \mathbf{H}_{\beta, \alpha, n}+\mathbf{N}$ as the measurement equation, and the transmitted signal $\mathbf{X}$ is the measurement matrix. But because we do not know about the transmitted signal, we may just employ the mean of the transmitted signal to be the measurement matrix of the proposed Kalman algorithm. We substitute $\overline{\mathbf{X}}=\mathbf{E}[\mathbf{X}]$ into $\mathbf{Y}=\overline{\mathbf{X}} \mathbf{H}_{\beta, \alpha, n}+\mathbf{N}$, then we serve the $\mathbf{N}$ as the measurement noise $\mathbf{v}_{n}$. Finally we can obtain a new measurement equation $\mathbf{Y}=\overline{\mathbf{X}} \mathbf{H}_{\beta, \alpha, n}+\mathbf{v}_{\boldsymbol{n}}$. The Kalman algorithm has two equations, the process equation and the measurement equation shown as follows,

$$
\begin{gathered}
\mathbf{H}_{\beta, \alpha, n+1}=\mathbf{F}_{n+1} \mathbf{H}_{\beta, \alpha, n}+\mathbf{p}_{n} \\
\mathbf{Y}=\mathbf{M}_{n} \mathbf{H}_{\beta, \alpha, n}+\mathbf{v}_{n}
\end{gathered}
$$




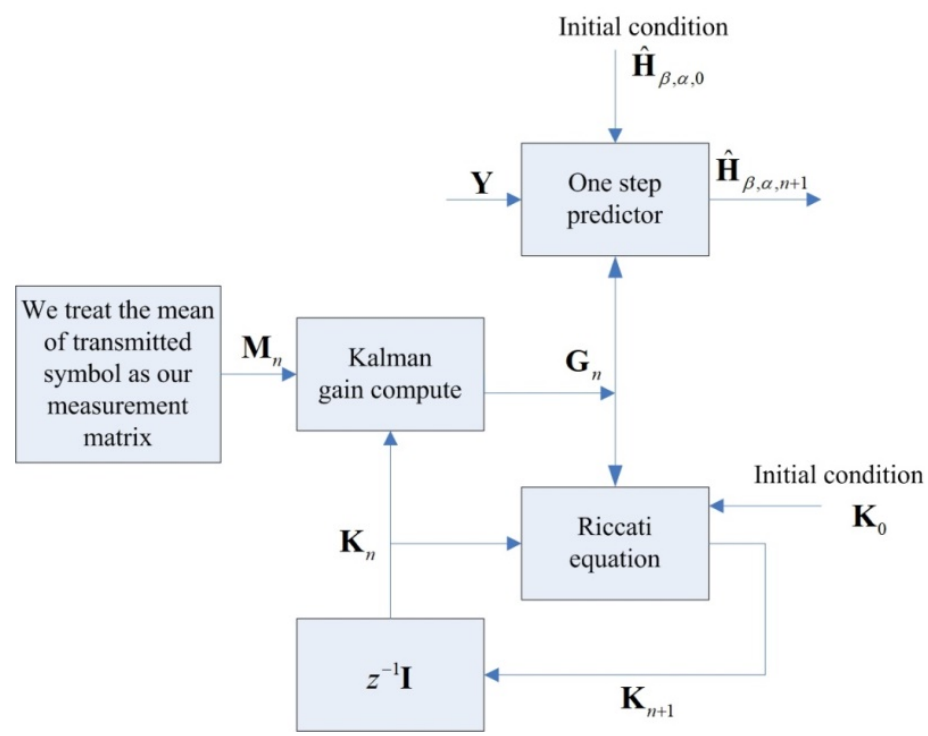

Fig. 3. Block diagram of Kalman channel estimation

The above measurement equation can be rewritten as follows,

$$
\widehat{\mathbf{Y}}=\mathbf{M}_{n} \widehat{\mathbf{H}}_{\beta, \alpha, n}+\mathbf{v}_{n}
$$

Based on the following assumptions

$$
\begin{aligned}
& E\left[\mathbf{p}_{n} \mathbf{p}_{m}^{H}\right]=\left\{\begin{array}{c}
\mathbf{p}_{n} \boldsymbol{n}=\boldsymbol{m} \\
\mathbf{0} \boldsymbol{n} \neq \boldsymbol{m}
\end{array}\right.
\end{aligned}
$$

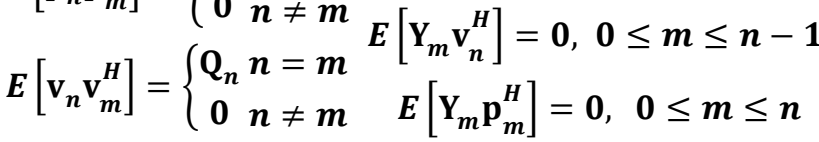

$$
\begin{aligned}
& E\left[\mathbf{p}_{n} \mathbf{v}_{m}^{H}\right]=\mathbf{0}
\end{aligned}
$$

The following estimate can be obtained

$$
\widehat{\mathbf{Y}}=\mathbf{M}_{n} \widehat{\mathbf{H}}_{\beta, \alpha, n}
$$

We further compute the innovation vector that can be described as follows,

So we obtain

$$
\alpha_{n}=\mathbf{Y}-\mathbf{M}_{n} \widehat{\mathbf{H}}_{\beta, \alpha, n}
$$

$$
\alpha_{n}=\mathbf{M}_{n} \mathbf{e}_{n}+\mathbf{v}_{n}
$$

where $\mathbf{e}_{\boldsymbol{n}}=\mathbf{H}_{\boldsymbol{\beta}, \boldsymbol{\alpha}, \boldsymbol{n}}-\widehat{\mathbf{H}}_{\boldsymbol{\beta}, \boldsymbol{\alpha}, \boldsymbol{n}}$ is the estimation error. This estimate can be expressed as a linear combination of the sequence of the innovation process. That is to say, we obtain

$$
\widehat{\mathbf{H}}_{\beta, \alpha, i}=\sum_{r=1}^{n} \mathbf{B}_{i, r} \alpha_{r}
$$

Using the assumption that $E\left[\mathbf{e}_{i, n} \alpha_{j}^{H}\right]=\mathbf{0}, j=1, \ldots, n$, and using $E\left[p_{n} p_{m}^{H}\right]=\left\{\begin{array}{c}\mathbf{R}_{n} n=m \\ 0 \quad n \neq m\end{array}\right.$, We can derive

$$
E\left[\mathbf{H}_{\beta, \alpha, i} \alpha_{j}^{H}\right]=\mathbf{B}_{i, j} \mathbf{R}_{j}
$$


Then, we multiply the inverse of $\mathbf{R}_{\boldsymbol{j}}$ for the both side of (42), and then we obtain

$$
\mathbf{B}_{i, j}=E\left[\mathbf{H}_{\beta, \alpha, i} \alpha_{j}^{H}\right]\left[\mathbf{R}_{j}\right]^{-1}
$$

Substituting (43) into (41), we can derive

$$
\widehat{\mathbf{H}}_{\beta, \alpha, i}=\sum_{r=1}^{n-1} E\left[\mathbf{H}_{\beta, \alpha, i} \alpha_{r}^{H}\right]\left[\mathbf{R}_{r}\right]^{-1} \alpha_{r}+E\left[\mathbf{H}_{\beta, \alpha, i} \alpha_{r}^{H}\right]\left[\mathbf{R}_{n}\right]^{-1} \alpha_{n}
$$

Setting $i=n+1$, the following equation can be derived

$$
\widehat{\mathbf{H}}_{\beta, \alpha, n+1}=\sum_{r=1}^{n-1} E\left[\mathbf{H}_{\beta, \alpha, n+1} \alpha_{r}^{H}\right]\left[\mathbf{R}_{r}^{(u)}\right]^{-1} \alpha_{r}+E\left[\mathbf{H}_{\beta, \alpha, n+1} \alpha_{r}^{H}\right]\left[\mathbf{R}_{n}\right]^{-1} \alpha_{n}
$$

The above equation indicate the relation between $\mathbf{H}_{\boldsymbol{\beta}, \boldsymbol{\alpha}, \boldsymbol{n + 1}}$ and $\mathbf{H}_{\boldsymbol{\beta}, \boldsymbol{\alpha}, \boldsymbol{n}}$, and we can derive the following equation

$$
E\left[\mathbf{H}_{\beta, \alpha, n+1} \alpha_{r}^{H}\right]=\mathbf{F}_{n+1} E\left[\mathbf{H}_{\beta, \alpha, n} \alpha_{r}^{H}\right]
$$

The summation term of (45) can be rewritten as follows,

Define the gain as follows,

$$
\sum_{r=1}^{n-1} E\left[\mathbf{H}_{\beta, \alpha, n+1} \alpha_{r}^{H}\right]\left[\mathbf{R}_{r}\right]^{-1} \alpha_{r}^{(u)}=\mathbf{F}_{n+1} \widehat{\mathbf{H}}_{\beta, \alpha, n}
$$

The following equations can be derived,

$$
\begin{gathered}
E\left[\mathbf{H}_{\beta, \alpha, n+1} \alpha_{n}^{H}\right]=\mathbf{F}_{n+1} E\left[\mathbf{H}_{\beta, \alpha, n} \alpha_{n}^{H}\right]=\mathbf{F}_{n+1} E\left[\mathbf{H}_{\beta, \alpha, n} \boldsymbol{e}_{n}^{H}\right] \mathbf{M}_{n}^{H} \\
E\left[\mathbf{H}_{\beta, \alpha, n+1} \alpha_{n}^{H}\right]=\mathbf{F}_{n+1} E\left[\boldsymbol{e}_{n} \boldsymbol{e}_{n}^{H}\right] \mathbf{M}_{n}^{H}=\mathbf{F}_{n+1} \mathbf{K}_{n} \mathbf{M}_{n}^{H}
\end{gathered}
$$

Finally, we obtain the gain as follows,

$$
\mathbf{G}_{n}=\mathbf{F}_{n+1} \mathbf{K}_{n} \mathbf{M}_{n}^{H}\left[\mathbf{R}_{n}\right]^{-1}
$$

where $\mathbf{K}_{n}=E\left[\boldsymbol{e}_{n} \boldsymbol{e}_{n}^{H}\right]$ is error correlation matrix, $\mathbf{R}_{n}=E\left[\alpha_{n} \alpha_{n}^{H}\right]$ is correlation matrix of innovation vector. After some manipulations, $\mathbf{R}_{n}$ can be derived as

$$
\mathbf{R}_{n}=\mathbf{M}_{n} \mathbf{K}_{n} \mathbf{M}_{n}^{H}+\mathbf{Q}_{n}
$$

Then, using the definition of the gain $\mathbf{G}_{\boldsymbol{n}}$, we can rewrite (45) as our estimation equation and can be expressed as follows,

$$
\widehat{\mathbf{H}}_{\beta, \alpha, n+1}=\mathbf{F}_{n+1} \widehat{\mathbf{H}}_{\beta, \alpha, n}+\mathbf{G}_{n} \alpha_{n}
$$

The following procedure is to derive the equation of the error correlation matrix. We know that

$$
\mathbf{e}_{n+1}=\mathbf{H}_{\beta, \alpha, n+1}-\widehat{\mathbf{H}}_{\beta, \alpha, n+1}
$$

So we can obtain

$$
\mathbf{e}_{n+1}=\mathbf{F}_{n+1}\left[\mathbf{H}_{\beta, \alpha, n}-\widehat{\mathbf{H}}_{\beta, \alpha, n}\right]-\mathbf{G}_{n}\left[\mathbf{Y}-\mathbf{M}_{n} \widehat{\mathbf{H}}_{\beta, \alpha, n}\right]+\mathbf{p}_{n}
$$

Next, based on (36), we get

$$
\mathbf{e}_{n}=\left[\mathbf{F}_{n+1}-\mathbf{G}_{n} \mathbf{M}_{n}\right] \mathbf{e}_{n}+\mathbf{p}_{n}-\mathbf{G}_{n} \mathbf{v}_{n}
$$


Substituting (56) into $\mathbf{K}_{n}=\boldsymbol{E}\left[\mathbf{e}_{n} \mathbf{e}_{n}^{H}\right]$, we obtain

$$
\mathbf{K}_{n+1}=\left[\mathbf{F}_{n+1}-\mathbf{G}_{n} \mathbf{M}_{n}\right] \mathbf{K}_{n}\left[\mathbf{F}_{n+1}-\mathbf{G}_{n} \mathbf{M}_{n}\right]^{H}+\mathbf{P}_{n}+\mathbf{G}_{n} \mathbf{Q}_{n} \mathbf{G}_{n}^{H}
$$

Finally, we employ the gain and the correlation matrix of the innovation vector to obtain the Riccati equation. So the estimated error correlation matrix can be computed as follows,

\subsection{Turbo equalization}

$$
\begin{aligned}
& \mathbf{K}_{n+1}=\mathbf{F}_{n+1} \mathbf{K}_{n+1} \mathbf{F}_{n+1}^{H}+\mathbf{P}_{n} \\
& \mathbf{K}_{n}=\mathbf{K}_{n-1}-\mathbf{F}_{n} \mathbf{G}_{n} \mathbf{M}_{n} \mathbf{K}_{n-1}
\end{aligned}
$$

The prior LLR $L\left(\mathbf{D}^{\left(n_{r}\right)}\right)$ is used for computing the mean and variance of the transmitted symbol described as follows, [32]-[35]

$$
\begin{gathered}
\overline{\mathbf{D}}^{\left(n_{r}\right)=} \sum_{X^{(u)} \in \beta} \mathbf{D}^{\left(n_{r}\right)} P\left(\mathbf{D}^{\left(n_{r}\right)}=D\right)=P\left(\mathbf{D}^{\left(n_{r}\right)}=+1\right)-P\left(\mathbf{D}^{\left(n_{r}\right)}=-1\right) \\
=\frac{e^{L\left(\mathbf{D}^{\left(n_{r}\right)}\right)}}{1+e^{L\left(\mathbf{D}^{\left(n_{r}\right)}\right)}}-\frac{1}{1+e^{L\left(\mathbf{D}^{\left(n_{r}\right)}\right)}}=\tanh \left(L\left(\mathbf{D}^{\left(n_{r}\right)}\right) / 2\right) \\
v=\sum_{X^{(u)} \in \beta}\left|\mathbf{D}^{\left(n_{r}\right)} \overline{\mathbf{D}}^{\left(n_{r}\right)}\right|^{2} p(D=D)=1-|\bar{D}|^{2}
\end{gathered}
$$

The mean and variance of the transmitted symbol and the output signal of the multiuser detector $\widetilde{\mathbf{Y}}_{u}$ are the input signal of the MMSE equalizer. The turbo equalizer then final output the estimate of the transmitted symbol and extrinsic LLR. As we know, the extrinsic LLR is equal to a posteriori LLR minus a prior LLR as follows,

$$
L_{E}(D)=\ln \frac{p(D=+1 \mid \widehat{D})}{p(D=-1 \mid \widehat{D})}-\ln \frac{p(D=+1)}{p(D=-1)}=\ln \frac{p(\widehat{D} \mid D=+1)}{p(\widehat{D} \mid D=-1)}
$$

Here for reducing the computational complexity, we assume that the probability density function (PDF) $p\left(\hat{X}^{(u)} \mid X^{(u)}=X\right)$ is Gaussian distribution with parameters

$$
p(\widehat{D} \mid D=D) \approx \varnothing\left(\left(\widehat{D}-\mu_{D}\right) / \sigma_{D}\right) / \sigma_{D}
$$

where $\mu_{D}=E(\widehat{D} \mid D=D)$ is the mean of the Gaussian distribution, $\sigma_{D}^{2}=\operatorname{Cov}(\widehat{D}, \widehat{D} \mid D=D)$ is the variance of the Gaussian distribution. In order to perform the exact implementation of the MMSE equalizer, we need to compute the extrinsic LLRs, the estimates of the transmitted symbols, and the coefficients of the equalizer. Then given the separated signal of each receiver antenna $\mathbf{Y}=\left[\begin{array}{llll}\widetilde{\mathbf{Y}}_{1} & \widetilde{\mathbf{Y}}_{2} & \ldots & \widetilde{\mathbf{Y}}_{N_{t}}\end{array}\right]^{T}$, the channel estimation matrix 
$\widehat{\mathbf{H}}_{n_{r}, n_{t}}=\left[\begin{array}{cccc}\widehat{\mathbf{H}}_{1,1} & \widehat{\mathbf{H}}_{1,2} & \ldots & \widehat{\mathbf{H}}_{1, N_{t}} \\ \widehat{\mathbf{H}}_{2,1} & \widehat{\mathbf{H}}_{2,2} & \cdots & \widehat{\mathbf{H}}_{2, N_{t}} \\ \vdots & \vdots & \ddots & \vdots \\ \widehat{\mathbf{H}}_{N_{r}, 1} & \widehat{\mathbf{H}}_{N_{r}, 2} & \cdots & \widehat{\mathbf{H}}_{N_{r}, N_{t}}\end{array}\right]$,

the mean matrix of the transmitted symbol $\overline{\mathbf{D}}=\left[\begin{array}{llll}\overline{\mathbf{D}}_{1} & \overline{\mathbf{D}}_{2} & \ldots & \overline{\mathbf{D}}_{N_{t}}\end{array}\right]^{T}$ and the variance matrix of the transmitted symbol $\mathbf{V}=\operatorname{Diag}\left(v_{1}, v_{2}, \ldots, v_{N_{t}}\right)$, the following equation

$$
\widehat{\mathbf{D}}=E(\mathbf{D})+\operatorname{Cov}(\mathbf{D}, \mathbf{Y}) \operatorname{Cov}(\mathbf{Y}, \mathbf{Y})^{-1}(\mathbf{Y}-E(\mathbf{Y}))
$$

It can be rewritten as follows,

$$
\widehat{\mathbf{D}}=\overline{\mathbf{D}}+\mathbf{V} \widetilde{\mathbf{H}}^{H}\left(\sigma_{w}^{2} \mathbf{I}+\widetilde{\mathbf{H}} \mathbf{V} \widetilde{\mathbf{H}}^{H}\right)^{-1}(\mathbf{Y}-\widetilde{\mathbf{H}} \overline{\mathbf{D}})
$$

Substituting the above equation into the MMSE equalization $\widehat{\mathbf{D}}=\sum \mathbf{C}(\mathbf{Y}-E(\mathbf{Y}))$, and then do partial deviation to the coefficients of the equalizer $\mathbf{C}$, we can get the equalizer as follows,

$$
\mathbf{C}=\left(\sigma_{w}^{2} \mathbf{I}+\widetilde{\mathbf{H}}^{(u)} \mathbf{V}^{(u)} \widetilde{\mathbf{H}}^{H}+\left(\mathbf{I}_{\text {diag }}-\mathbf{V}\right)\right)^{-1} \widetilde{\mathbf{H}}
$$

The above equation can also be used to compute the mean and variance of the transmitted symbol as follows,

$$
\begin{aligned}
\mu_{D}=E(\widehat{D} \mid D=D) & =D \mathbf{C}^{H} \\
\sigma_{D}^{2}=\operatorname{Cov}(\widehat{D}, \widehat{D} \mid D=D) & =\mathbf{C}^{H}(\mathbf{I}-\mathbf{C})
\end{aligned}
$$

We then find the following equation to get the Gaussian distribution described above,

$$
L_{E}(\mathbf{D})=\ln \frac{\emptyset\left(\left(\widehat{\mathbf{D}}-\mu_{+1}\right) / \sigma_{+1}\right) / \sigma_{+1}}{\emptyset\left(\left(\widehat{\mathbf{D}}-\mu_{-1}\right) / \sigma_{-1}\right) / \sigma_{-1}}=\frac{2 \widehat{\mathbf{D}} \mu_{+1}}{\sigma_{+1}^{2}}=2 \mathbf{C}^{H}(\mathbf{Y}-\widetilde{\mathbf{H}} \overline{\mathbf{D}}) /(\mathbf{I}-\mathbf{C})
$$

The extrinsic LLR is used for the decoder. For initial step, the a priori information is zero. We substitute this condition into the above equations and the initial values can be described as follows,

$$
\begin{gathered}
\widehat{\mathbf{D}}=\widetilde{\mathbf{H}}^{H}\left(\sigma_{w}^{2} \mathbf{I}+\widetilde{\mathbf{H}} \widetilde{\mathbf{H}}^{H}\right)^{-1} \mathbf{Y} \\
\mathbf{C}_{N A}=\left.\left(\sigma_{w}^{2} \mathbf{I}+\widetilde{\mathbf{H}} \mathbf{V} \widetilde{\mathbf{H}}^{H}+\left(\mathbf{I}_{\text {diag }}-\mathbf{V}\right)\right)^{-1} \widetilde{\mathbf{H}}\right|_{L(\mathbf{D})=0}=\left(\sigma_{w}^{2} \mathbf{I}+\widetilde{\mathbf{H}} \widetilde{\mathbf{H}}^{H}\right)^{-1} \widetilde{\mathbf{H}} \\
L_{E}(\mathbf{D})=2 \mathbf{C}_{N A}^{H} \mathbf{Y} /\left(\mathbf{I}-\mathbf{C}_{N A}\right)
\end{gathered}
$$




\section{Simulation Results}

In this section, we compare the performances of existing schemes (CC, PRCC, Two stage IQ-Imbalance schemes), optimal solution (known CFO and known channel state information) and the proposed turbo receiver. The adopted channel models are multipath Rayleigh fading and ITU channel models defined in the IEEE 802.11 Working Group [33]. We simulate those schemes in the multipath Rayleigh fading channel model and ITU channel model for vehicular test environments with 6 path taps. In these systems, we use QPSK modulation and 16QAM modulation for MIMO-OFDM systems, and then we apply two antennas at transmitter, and two antennas at receiver. The simulated MIMO-OFDM systems assume there are 256 subcarriers (i.e., FFT size $=256$ ), 20 cyclic prefixes (i.e., $\mathrm{CP}=20$ ), difference of speed(i.e., $\mathrm{V}=5$, $60,100,200,300 \mathrm{~km} / \mathrm{hr}$ ), and different frequency offset (i.e., $\varepsilon=0,0.15,0.25$ ). The turbo encoder employs convolution code, and the code rate is $1 / 2$. The turbo decoder employs MAP decoder.

Fig. 4 shows the BER performance with different iteration for the proposed turbo receiver. The signal format is QPSK modulation, CFO $\varepsilon=0.15$ and the channel is multipath channel. In this figure, we can see that the performances with third and fourth iterations are almost the same, so the third iteration can be chosen in the proposed turbo receiver. Fig. 5 shows the BER performance with different iteration in the proposed turbo receiver. The signal format is QPSK modulation, $\mathrm{CFO} \varepsilon=0.25$ and the channel is multipath channel model. We can see that the performances with third and fourth iterations are almost the same, so the third iteration can be chosen in the proposed turbo receiver. Fig. 6 shows the BER comparison of the no CFO Solution, CC, PRCC, IQ Imbalance, the optimal scheme, the adaptive modified PRCC schemes and the proposed turbo receiver with QPSK modulation and $\varepsilon=0.15$ under ITU Channel A of $100 \mathrm{~km} / \mathrm{hr}$. It demonstrates that the performance of the proposed turbo modified receiver is better than those of CC, PRCC and IQ imbalance. The turbo receiver with modified PRCC improve the performance for about $5 \mathrm{~dB}$. Fig. 7 shows the BER comparison of the no CFO solution, CC, PRCC, IQ Imbalance, the optimal scheme, the adaptive modified PRCC scheme and the proposed turbo receiver with QPSK modulation, $\varepsilon=0.25$ under ITU Channel A with speed $100 \mathrm{~km} / \mathrm{hr}$. It demonstrates that the performance of the proposed turbo receiver is better than those of CC, PRCC and IQ imbalance. This is because that the developed turbo receiver can track CFOs and update the phase rotations based on the estimated CFOs from the two paths in fast fading channel. 


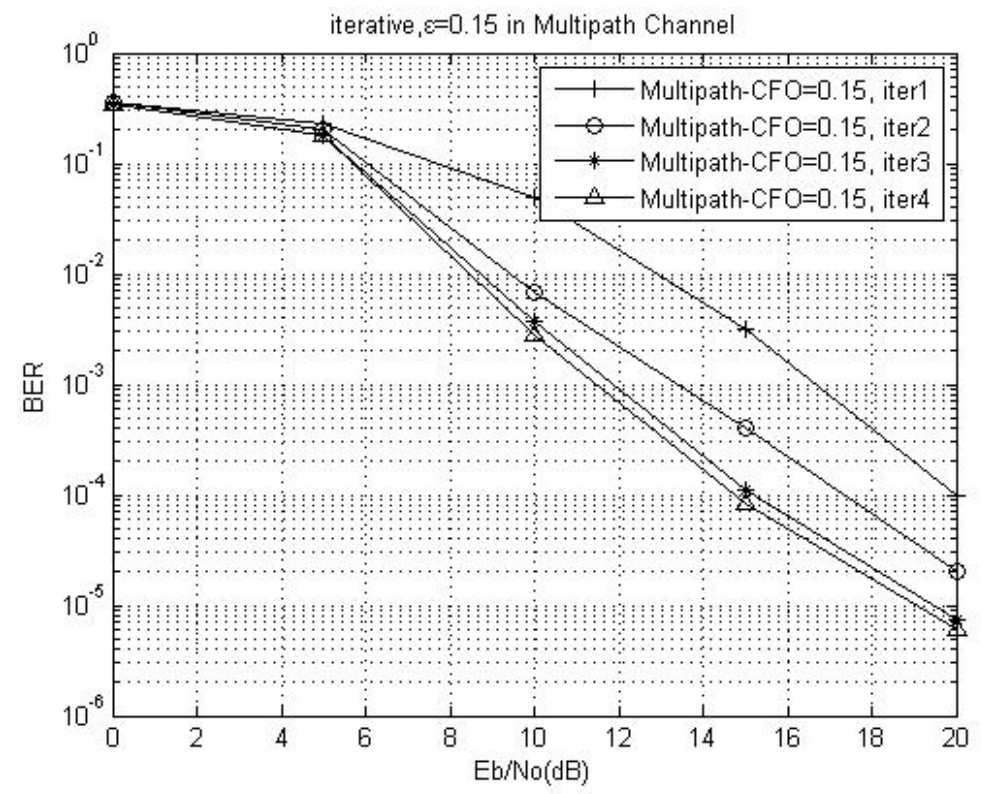

Fig. 4. The BER performance of different iteration for the proposed receiver with QPSK in multipath channel model, $\mathrm{CFO}=0.15$.

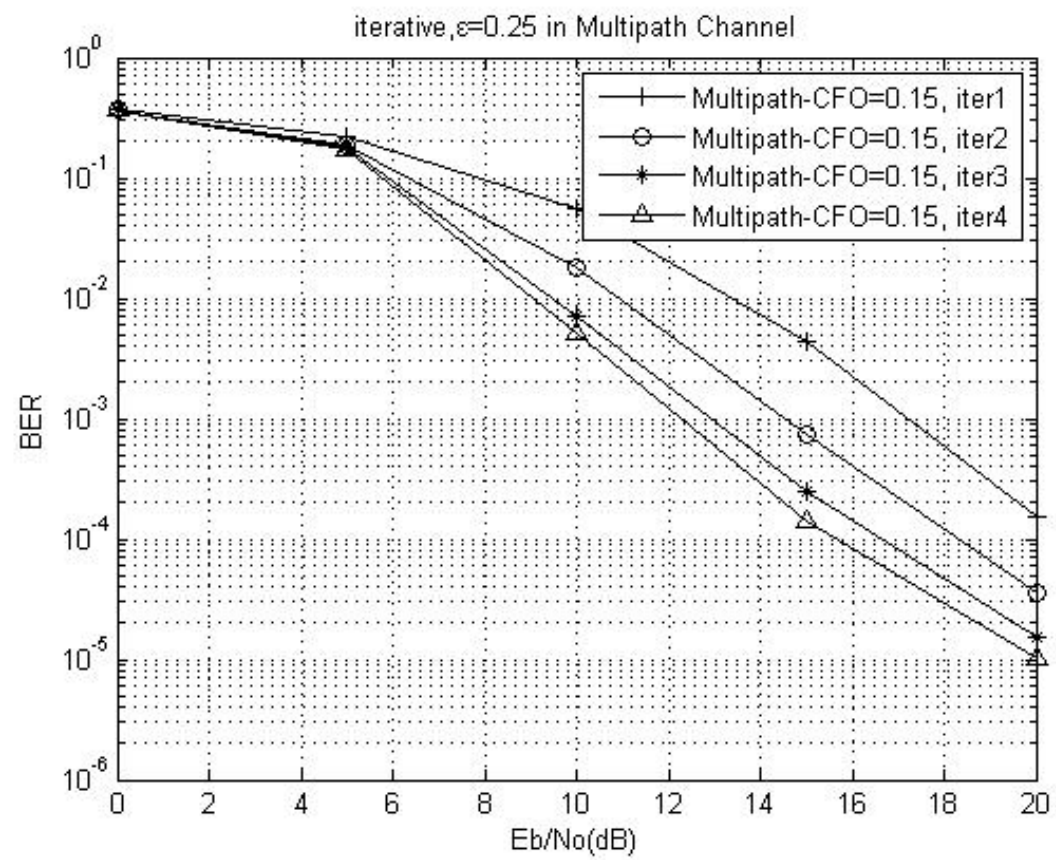

Fig. 5. The BER performance of different iteration for the proposed receiver with QPSK in multipath channel model, $\mathrm{CFO}=0.25$. 


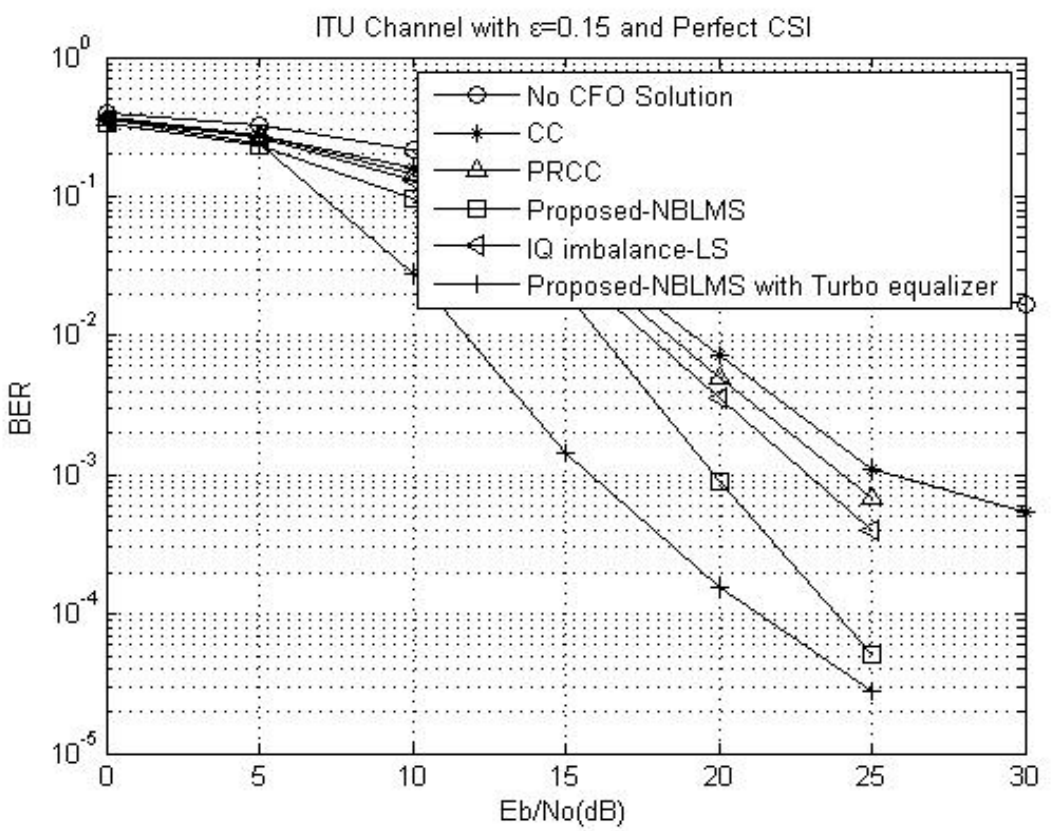

Fig. 6. The BER comparisons of existing methods and the proposed turbo receiver, $\varepsilon=0.15$ under ITU

Channel A of $100 \mathrm{~km} / \mathrm{hr}$.

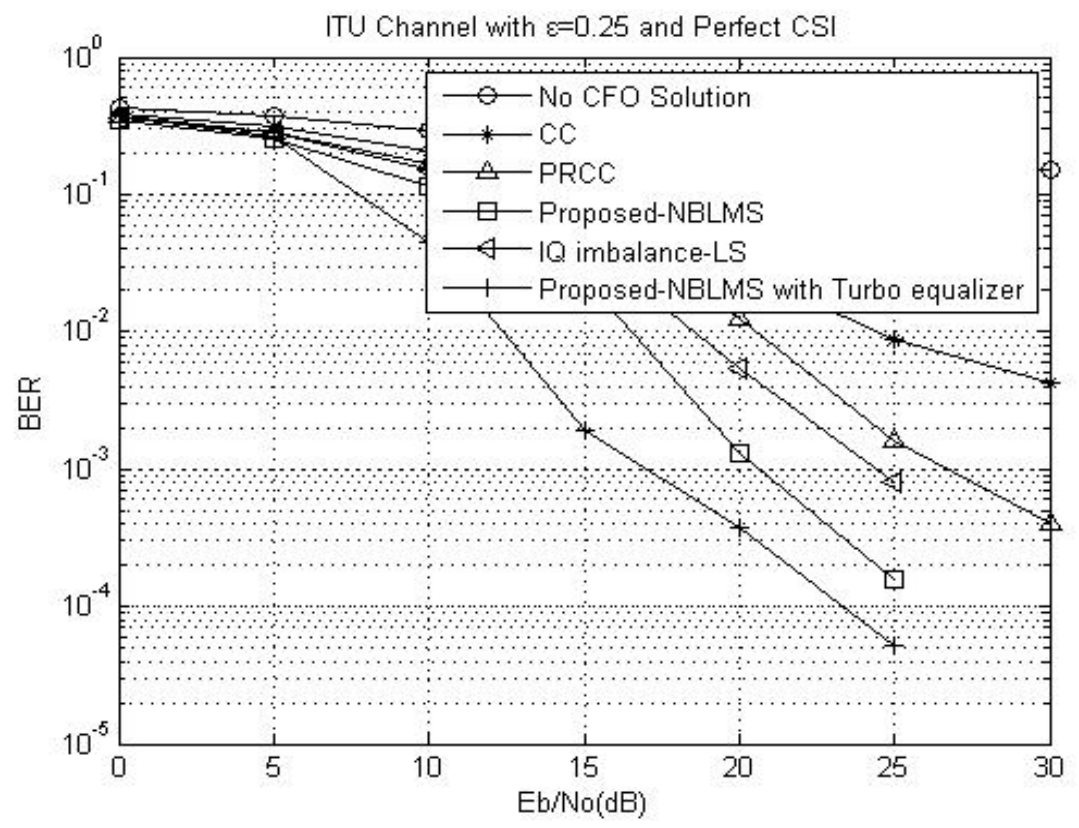

Fig. 7. The BER comparisons of existing methods and the proposed turbo receiver, $\varepsilon=0.25$ under ITU Channel A of $100 \mathrm{~km} / \mathrm{hr}$.

Fig. 8 shows the BER comparison of the no CFO solution, CC, PRCC, IQ Imbalance, the optimal scheme and the proposed turbo receiver with QPSK modulation, $\varepsilon=0.15$ in multipath fading channel with speed $100 \mathrm{~km} / \mathrm{hr}$. It demonstrates that the performance of the proposed turbo receiver is better than those of CC, PRCC and IQ imbalance. We can see that the excess 
delay of ITU channel A is larger than the excess delay of multipath fading channel, and then the coherence bandwidth of multipath fading channel is larger than the coherence bandwidth of ITU channel A, so the performance affected by the multipath fading channel is lower than that by ITU channel A. Fig. 9 shows the BER comparison of the no CFO solution, CC, PRCC, IQ Imbalance, the optimal scheme and the proposed turbo receiver with QPSK modulation, $\varepsilon=0.25$ in multipath fading channel with speed $100 \mathrm{~km} / \mathrm{hr}$. It demonstrates that the performance of the proposed turbo receiver better than those of CC, PRCC and IQ imbalance.

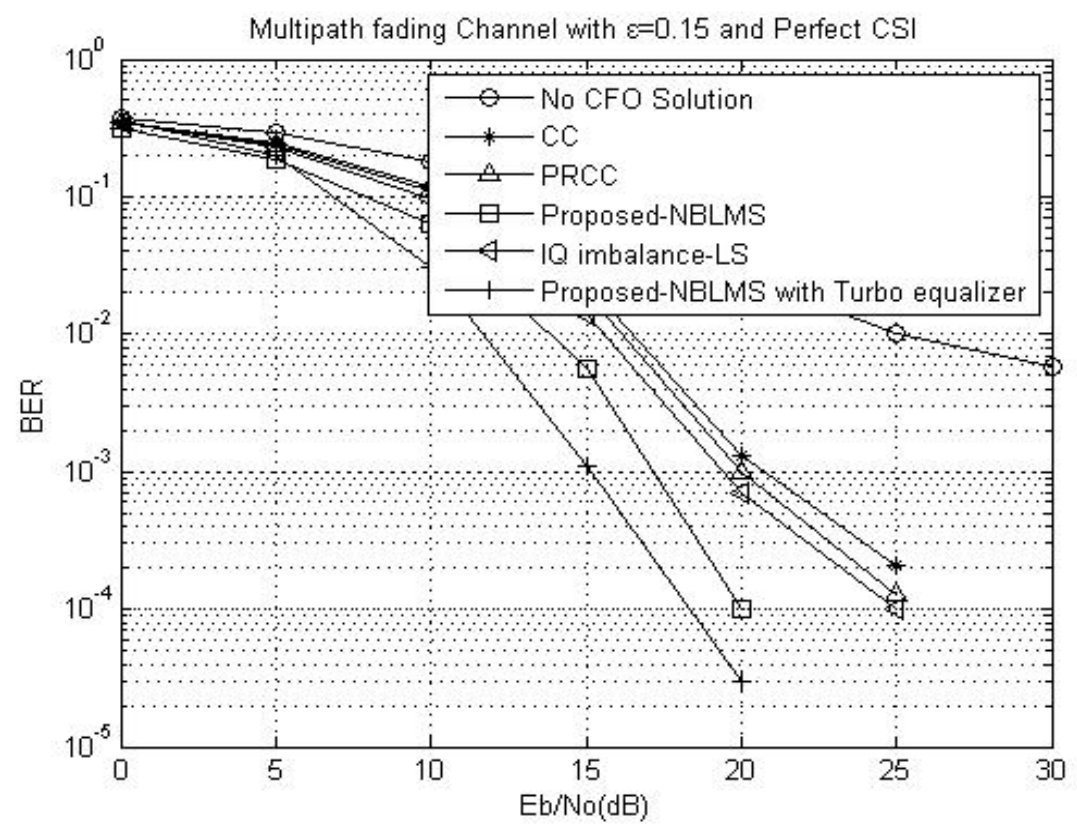

Fig. 8. The BER comparisons of existing methods and the proposed turbo receiver, $\varepsilon=0.15$ under multipath fading channel of $100 \mathrm{~km} / \mathrm{hr}$.

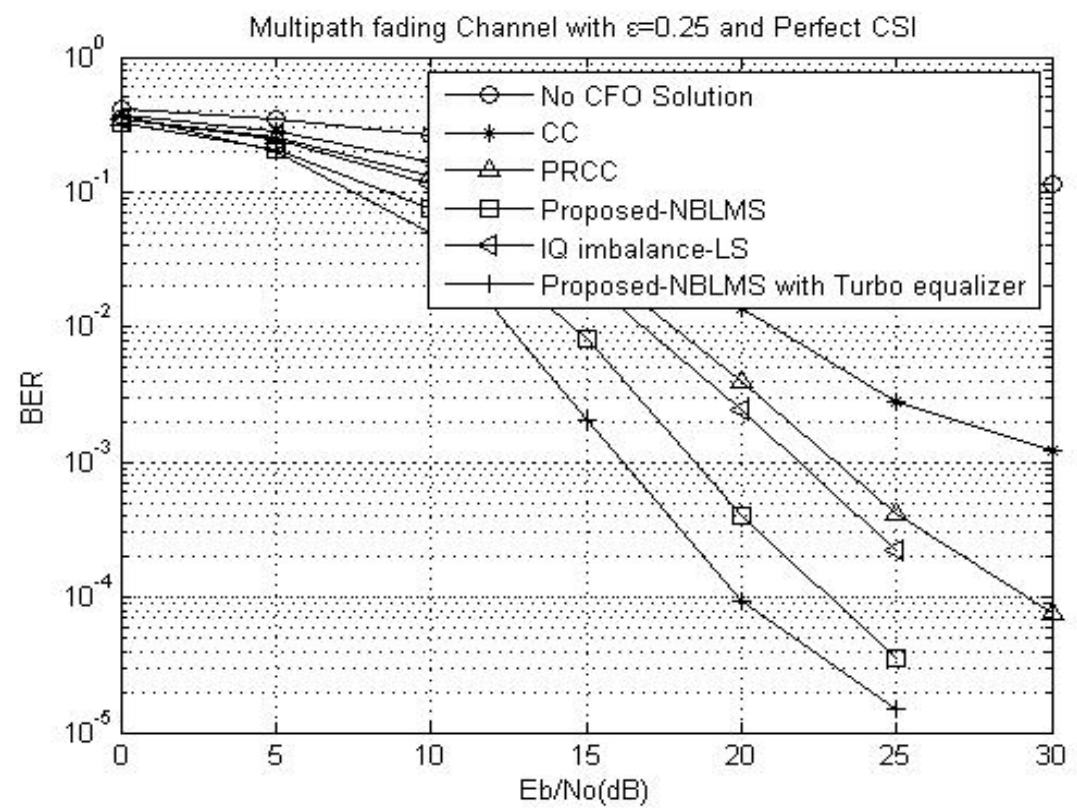

Fig. 9. The BER comparisons of existing methods and the proposed turbo receiver, $\varepsilon=0.25$ under multipath fading channel of $100 \mathrm{~km} / \mathrm{hr}$. 
Fig. 10 shows the BER comparison of the no CFO solution, CC, PRCC, IQ Imbalance, the optimal scheme and the proposed turbo receiver with QPSK modulation, $\varepsilon=0.25$ in multipath fading channel with speed $100 \mathrm{~km} / \mathrm{hr}$. The Kalman channel estimation is applied. It demonstrates that the performance of the proposed turbo receiver is better than those of CC, PRCC and IQ imbalance schemes. This is because the developed turbo receiver can track CFOs and update the phase rotations based on the estimated CFOs from the two paths in fast fading channel. Fig. 11 shows the BER comparison of the existing PRCC scheme and the proposed scheme at different speed with OPSK modulation and $\varepsilon=0.25$ at $\mathrm{SNR}=25 \mathrm{~dB}$. We can see that the proposed scheme outperforms the existing PRCC scheme at different speed. This difference is due to the proposed scheme can track CFOs and update the phase rotations, and the existing PRCC scheme can't bear the large CFO in fast speed.

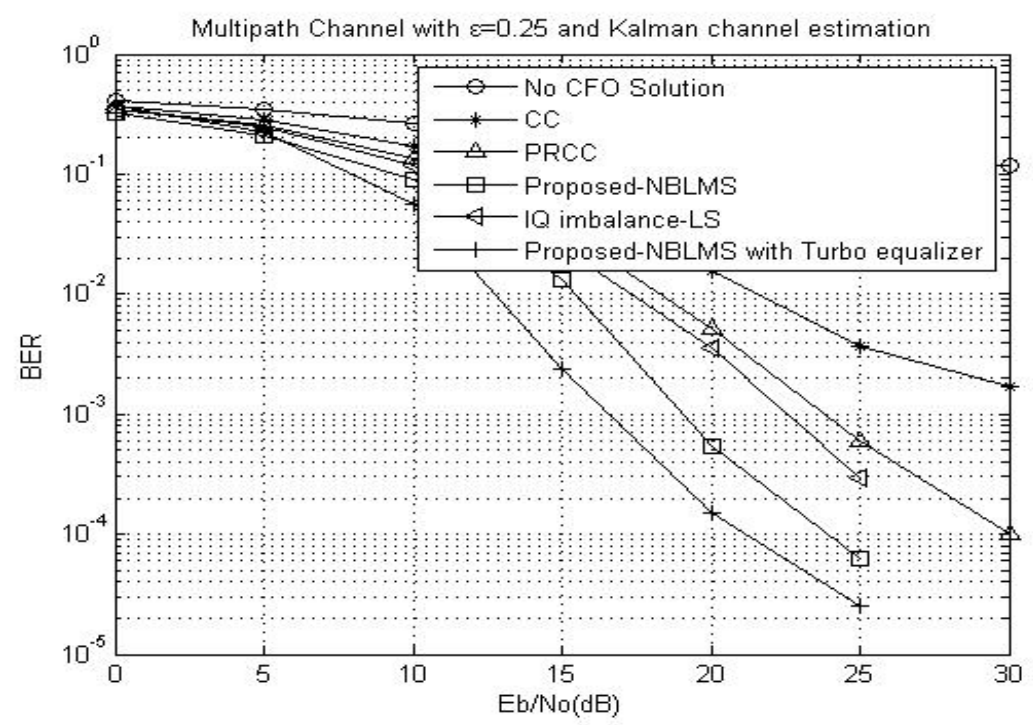

Fig. 10. The BER comparisons of existing methods and the proposed turbo receiver, $\varepsilon=0.25$ under Multipath Fading Channel of $100 \mathrm{~km} / \mathrm{hr}$ with Kalman channel estimation.

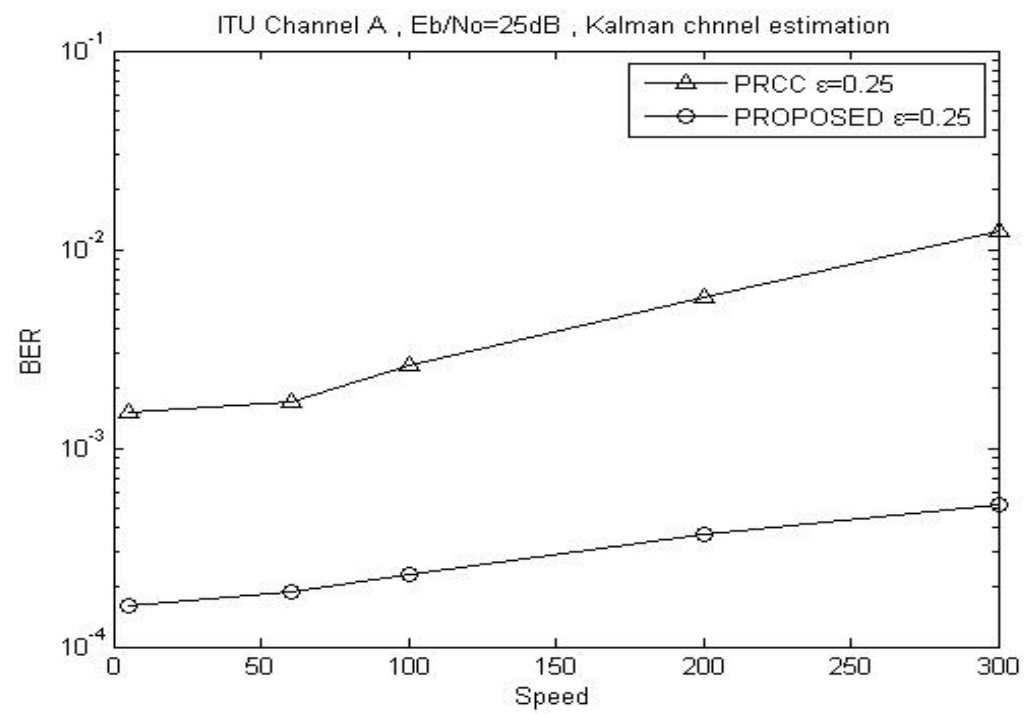

Fig. 11. The BER comparisons the proposed scheme and the existing PRCC scheme under different speed for $\varepsilon=0.25$ with QPSK modulation and kalman channel estimation at $\mathrm{SNR}=25 \mathrm{~dB}$. 


\section{Conclusion}

This paper proposes an advanced turbo receiver with ICI self cancellation, Kalman channel estimation and turbo equalization for MIMO-OFDM systems in time-varying channels. We employ an adaptive receiver and construct two-path conjugate transmission that is based on the PRCC concept. Additionally, the Kalman filter can efficiently track the time-varying channels. We also use iterative property of turbo equalization to update the Kalman filter, equalizer and decoder to improve the receiver performance. With such adaptive phase rotations in the receiver, the CFO variations due to the channel effect and the mismatch between oscillators at the transmitter and receiver can effectively be tracked without feeding back the CFO estimate to the transmitter as required in conventional PRCC scheme.

\section{References}

[1] S. M. Alamouti, "A simple transmit diversity technique for wireless communications," IEEE J. Select. Areas Commun., vol. 16, no. 8, pp. 1451-1458, 1998.Article (CrossRef Link)

[2] R. Zayani, R. Bouallegue and D. Roviras, "Adaptive Pre-distortions Based on Neural Networks Associated with Levenberg-Marquardt Algorithm for Satellite Down Links," EURASIP Journal on Wireless Communications and Networking, vol. 2008, pp. 1-8, 2008.Article (CrossRef Link)

[3] A. J. Goldsmith, S. A. Jafar, N. Jindal and S. Vishwanath, "Capacity Limits of MIMO Channels," IEEE Journal on Selected Areas in Communication, vol. 21, no. 5, pp. 684-702, Sep. 2006. Article (CrossRef Link)

[4] J. Harshan and E. Viterbo, "Integer Space-Time Block Codes for Practical MIMO Systems," IEEE Tran. On Communication, vol. 2, no.4, pp. 455-458, Aug. 2013.Article (CrossRef Link)

[5] D. Hu, X. Wang and L. He, "A New Sparse Channel Estimation and Tracking Method for Time-Varying OFDM Systems," IEEE Trans. Commun., vol. 62, No. 9, pp. 4648-4653, Nov. 2013.Article (CrossRef Link)

[6] T.-L. Liu and W.-H. Chung, "ICI Self-Cancellation With Cosine Windowing in OFDM Transmitters Over Fast Time-Varying Channels," IEEE Trans. Commun., vol.14, no.7, pp. 3559-3570, July 2015.Article (CrossRef Link)

[7] H. Zareian and V. T. Vakili, "Analytical EVM, BER, and TD Performances of the OFDM Systems in the Presence of Jointly Nonlinear Distortion and IQ Imbalance," Ann. Telecommun., vol. 64, pp. 753-762, 2009. Article (CrossRef Link)

[8] S. A. Bassam, M. Helaoui and F. M. Ghannouchi, "Crossover Digital Predistorter for the Compensation of Crosstalk and Nonlinearity in MIMO Transmitters," IEEE Trans. Microwave T heory Tech., vol. 57, no. 5, pp. 1119-1128, May 2009. Article (CrossRef Link)

[9] T. C. W. Schenk and A. V. Zelst, "Frequency synchronization for MIMO-OFDM wireless LAN systems," in Proc. of IEEE 58 ${ }^{\text {th }}$ Vehicular Technology Conference, Volume: 2 , Page(s):781 785 , 6-9 Oct. 2003. Article (CrossRef Link)

[10] H. K. Shah, K. S. Dasgupta and H. Soni, "Low complexity scheme for carrier frequency offset estimation in orthogonal frequency division multiple access uplink,” IET Comm. vol. 7, Iss. 13, pp. 1405-1411, May. 2013 Article (CrossRef Link)

[11] C. P. del Amo and M. J. Fernández-Getino García, "Iterative Joint Estimation Procedure for Channel and Frequency Offset in Multi-Antenna OFDM Systems With an Insufficient Cyclic Prefix,” IEEE Trans. Veh. Tech. vol. 62, no. 8, pp. 3653-3662, Oct. 2013. Article (CrossRef Link)

[12] W.-S. Hou and B.-S. Chen, "ICI cancellation for OFDM communication systems in time varying multipath fading channels,” IEEE Trans. Wireless Commun., vol. 4, no. 5, pp. 2100-2110, Sep. 2005. Article (CrossRef Link)

[13] S. Lu and N. Al-Dhahir, "Coherent and differential ICI cancellation for mobile OFDM with application to DVB-H,” IEEE Trans. Wireless Commun., vol. 7, no. 11, pp. 4110-4116, July 2008. Article (CrossRef Link) 
[14] Y. Mostofi and D. C. Cox, “ICI mitigation for pilot-aided OFDM mobile systems,” IEEE Trans. Wireless Commun., vol. 4, no. 2, pp. 765-774, Mar. 2005. Article (CrossRef Link)

[15] H.-G. Yeh, Y.-K. Chang, and B. Hassibi, "A scheme for cancelling intercarrier interference using conjugate transmission in multicarrier communication systems," IEEE Trans. Wireless Commun., vol. 6, no. 1, pp. 3-7, Jan. 2007. Article (CrossRef Link)

[16] C.-L. Wang and Y.-C. Huang, "Intercarrier interference cancellation using general phase rotated conjugate transmission for OFDM systems,” IEEE Trans. Commun., vol. 58, no. 3, pp. 812-819, Mar. 2010. Article (CrossRef Link)

[17] C.-C. Wu, P. Ma, W.-D. Hung and C.-H. Kuo, "Two-stage Compensation for Non-ideal Effects in MIMO-OFDM Systems,” in Proc. IEEE APSIPA, Dec. 2012. Article (CrossRef Link)

[18] C.-L. Wang, P.-C. Shen, Y.-C. Lin and J.-H. Huang, “An Adaptive Receiver Design for OFDM Systems Using Conjugate Transmission,” IEEE Trans. Commun., Vol. 61, No. 2, pp. 599-608, Feb. 2013. Article (CrossRef Link)

[19] P. M. Clarkson, Optimal and Adaptive Signal Processing. CRC Press, page 203, 1993. Article (CrossRef Link)

[20] S. Haykin, Adaptive Filter Theory, 5th edition, Pearson, page 307, 2013. Article (CrossRef Link)

[21] S. M. Shah, R. Samar, M. A. Z. Raja and J. A. Chambers, "Fractional normalised filtered-error least mean squares algorithm for application in active noise control systems," Electronics Letters, vol. 50, No. 14, pp. 973-975, July 2014. Article (CrossRef Link)

[22] S. Noh, Y. Sung and M. D. Zoltowski, "A New Precoder Design for Blind Channel Estimation in MIMO-OFDM Systems,” IEEE Trans. Wireless Comm. , vol. 13, no. 12, pp. 7011-7024, Dec. 2014. Article (CrossRef Link)

[23] S. Park, B. Shim and J. W. Choi, "Iterative Channel Estimation Using Virtual Pilot Signals for MIMO-OFDM Systems,” IEEE Trans. Sig. Proc., vol. 63, no. 12, pp. 3032-3045, June. 2015. Article (CrossRef Link)

[24] M. Huang, X. Chen, L. Xiao, S. Zhou and J. Wang "Kalman-filter-based channel estimation for orthogonal frequency-division multiplexing systems in time-varying channels," IET Commun., Vol. 1, No. 4, pp. 795-801, Aug. 2007. Article (CrossRef Link)

[25] E. P. Simon and M. A. Khalighi, "Iterative Soft-Kalman Channel Estimation for Fast Time-Varying MIMO-OFDM Channels,” IEEE Wireless Comm. Letters, vol. 2, no. 6, pp. 599-602, Dec. 2013. Article (CrossRef Link)

[26] J.-H. Park, Y. Whang and K. S. Kim, "Low Complexity MMSE-SIC Equalizer Employing Time-Domain Recursion for OFDM Systems,” IEEE Sig. Proc. Letters, vol. 15, pp.633-636 2008. Article (CrossRef Link)

[27] X. Li and T. F. Wong, "Turbo equalization with nonlinear Kalman filtering for time-varying frequency-selective fading channels” IEEE Trans. Wireless Communications, pp. 691 - 700, on year 2007 Article (CrossRef Link)

[28] K. Kim, N. Kalantarova, S. S. Kozat and A. C. Singer,"Linear MMSE-Optimal Turbo Equalization Using Context Trees,” IEEE Trans. Signal Processing, Vol. 61, No. 12, pp. 3041-3055, Jun. 15, 2013 Article (CrossRef Link)

[29] L. Dong, "Turbo Equalization with Prediction and Iterative Estimation of Time-Varying Frequency-Selective Channels,” Wireless Personal Communications, vol. 55, no. 4, Dec. 2010. Article (CrossRef Link)

[30] W. Yaun, N. Wu, H. Wang and J. Kuang," Variational Inference-Based Frequency-Domain Equalization for Faster-Than-Nyquist Signaling in Doubly Selective Channels,"IEEE Signal Proc. Letter, Vol. 23, No. 9, Sep. 2016. Article (CrossRef Link)

[31] N. Wu, W. Yuan, H. Wang, Q. Shi and J. Kuang, "Frequency-Domain Iterative Message Passing Receiver for Faster-Than-Nyquist Signaling in Doubly Selective Channels,” IEEE Wireless Comm. Letter, Vol. 5, No. 6, June 2016. Article (CrossRef Link)

[32] S. Lin, and D. J. Costello, Error Control Coding, Pearson Prentice Hall, page 167, 2004. Article (CrossRef Link) 
[33] A. Tomasoni, M. Ferrari, D. Gatti, F. Osnato and S. Bellini, “A Low Complexity Turbo MMSE Receiver for W-LAN MIMO Systems," in Proc. of IEEE International Conference on Communications, vol.9, pp. 4119-4124, June 2006. Article (CrossRef Link)

[34] S. Talakoub, L. Sabeti, B. Shahrrava and M. Ahmadi, “An Improved Max-Log-MAP Algorithm for Turbo Decoding and Turbo Equalization,” IEEE Trans. Instrumentation and Measurement, vol.56, no.3, pp.1058-1063, June 2007. Article (CrossRef Link)

[35] B. O'Hara and A. Petrick, The IEEE 802.11 Handbook: A Designer's Companion, $2^{\text {th }}$ edition, page 67, 2011. Article (CrossRef Link)

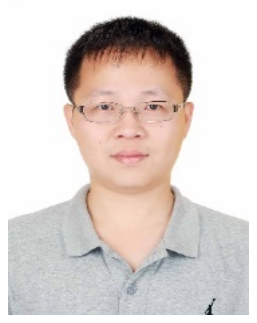

Yu-Kuan Chang was born in Kaohsiung, Taiwan, in 1986. He received the B.Sc. degree in aeronautical engineering from National Formosa University (NFU), Yunlin, Taiwan, in 2008 and the M.Sc. degree from the National Chi Nan University (NCNU), Nantou, Taiwan, in 2010. He is a member of the Communications System Laboratory, National Chung Hsing University (NCHU). His research interest is focused on MIMO MC-CDMA and LTE communication systems.

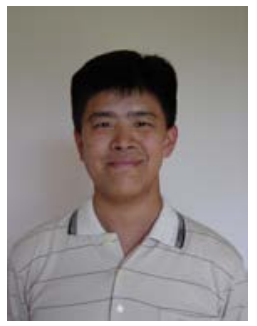

Fang-Biau Ueng received the Ph.D. degree in electronic engineering from the National Chiao Tung University, Hsinchu, Taiwan in 1995. From 1996 to 2001 he was with National Space Program Office (NSPO) of Taiwan as an associate researcher. From 2001 to 2002 he was with Siemens Telecommunication Systems Limited (STSL), Taipei, Taiwan, where he was involved in the design of mobile communication systems. Since February 2002 he has been with the department of electrical engineering, National Chung-Hsing University, Taichung, Taiwan. His areas of research interest are wireless communication and adaptive signal processing.

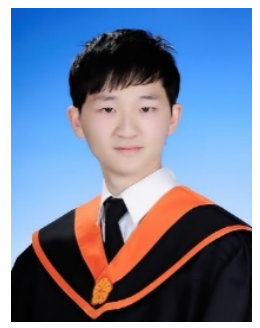

Yi-Wei Jhang was born in Miaoli, Taiwan, on September 1990. He received the B.S. degree from the Department of communication Engineering, Chung Hua University (CHU), Hsinchu, Taiwan, in 2013, and M.S. degree in communication engineering from the National Chung Hsing University (NCHU), Taichung, Taiwan, in 2015. 